\title{
Prospective Study to Identify Commonest Organisms and Antibiotic Sensitivity in Peritonitis Due to Duodenal Ulcer Perforation in Govt. Rajaji Hospital
}

\author{
Dr.C.Gangalaksmi ${ }^{1}$, Dr.R.Tennison ${ }^{2}$ \\ ${ }^{1}$ Assistant Professor, Department of General Surgery, Madurai Medical College, Madurai, Tamilnadu, India. \\ ${ }^{2}$ Post Graduate, Department of General Surgery, Madurai Medical College, Madurai, Tamilnadu, India.
}

\begin{abstract}
Perforative peritonitis is one of the most commonly encountered surgical acute abdominal emergencies in surgery casuality. The importance of this study is to reduce the postoperative complications like septicemia, wound infection, burst abdomen, bronchopneumonia, long hospital stay etc., by identifying the inciting organisms and its sensitive antibiotics rather than using empirical antibiotic therapy which is followed at present, in immediate postoperative period. Among hollow viscus perforations duodenal perforation is the most common site. The organisms setting up the peritonitis features are diverse according to the location of perforation. The common organisms isolated in proximal bowel perforation are E.coli, klebsiella, lactobacilli, streptococci, candida. There is an increasing trend in isolating anaerobic organisms or polymicrobial isolates as the perforation occurs distally and the time interval between the disease onset and time of intervention increases. Majority of rural patients present in surgical opd lately. Also there is a direct relation between interval of disease onset \& time of intervention and postoperative morbidity. In proximal bowel perforation, proposed antibiotic coverage is against gram positive cocci and gram negative enteric organisms. Hence administration of appropriate antibiotics in immediate postoperative period reduces poor postoperative outcome.
\end{abstract}

KEYWORDS: Duodenal ulcer, perforative peritonitis.

\section{Introduction}

Perforative peritonitis is one of the most commonly encountered surgical emergencies in all setups for a practicing surgeon. Among all hollow viscus perforative peritonitis, DU perforation is the most common cause of perforative peritonitis. In spite of easy diagnosis, operative procedures and advent of newer antibiotics, postoperative morbidity and mortality is still a challenging task for all surgeons. Postoperative complications like wound infection, intraabdominal infection, septicemia and mortality can be easily combated better with appropriate sensitive antibiotics than empirical antibiotic policy. Though many researches clearly depicting that there are only few common groups of organisms being isolated, sensitivity pattern differs in different setups. Hence sensitive appropriate postoperative antibiotic policy plays an important additive role to surgical treatment in managing perforative peritonitis.

Aim of the study: To study the bacteriological profile in the peritonitis patient due to duodenal ulcer perforation and their sensitive antibiotics by collecting peritoneal fluid and to reduce postoperative morbidity and mortality .

\section{Objectives of the study:}

A. To detect commonest organisms in peritonitis due to duodenal ulcer perforation.

B. To find out antibiotic sensitivity pattern in peritonitis due to duodenal ulcer perforation.

C. To see response of patients after starting antibiotics according to the culture and sensitivity report in terms postoperative complications like septicemia, surgical site infection and hospital stay.

\section{Eligibility criteria:}

A.Inclusion Criteria:

1. All peritonitis patients with duodenal ulcer perforation.

2. Patient's age above 13yrs.

3. Patients with stable vitals who are fit for surgery.

4. Patients consented for inclusion in the study

\section{B.Exclusion Criteria:}

1. Patients with peritonitis due to other hollow viscus perforation. 
2. Patient's age below 13 yrs.

3. Patients with traumatic perforation

4. Patients with perforative peritonitis not operated due to unstable vitals.

5. Patients in immunocompromised state like HIV, TB.

\section{Materials Used:}

1. Proforma containing patient history, clinical examination, investigations, intraoperative findings and operative notes.

2. Informed consent forms.

3. Sterile container with screw cap and requisition forms.

\section{Methodology:}

This study is designed to know the bacteriological profile and their sensitive antibiotic for the peritonitis patients.

- 140 patients who are admitted in GRH between JAN,2016 and AUG,2016 with bowel perforation are studied

- Intra operative peritoneal toxic fluid culture and sensitivity is done in all patients taken up for surgery

- Effectiveness in view of post-op outcome is studied between empirical versus specific antibiotic administration.

- Then the study proceeds to identify the bacteriological profile, sensitive antibiotics and compare the postop outcomes between administration of empirical versus specific antibiotic pattern.

Collection of sample and transport:

- Peritoneal toxic fluid is collected after opening the peritoneum

- $5 \mathrm{ml}$ of fluid will be collected in sterile screw cap container

- Sample will be transported from OT to microbiology lab at room temperature immediately if possible. Sample stored in refrigerator at 6degree celcius if transportation delayed for more than 3 hours

\section{Processing of sample:}

- $1^{\text {st }}$ day: microbiology and culture inoculation.

- $2^{\text {nd }}$ day: if growth is present then organisms are identified, then antibiotic sensitivity test is performed

- $\quad 3^{\text {rd }}$ day: Isolated organisms and sensitive antibiotic is ready...

\section{Assessment of post-operative outcomes:}

1. Postoperative outcomes are assessed in terms of postoperative complications like

$\checkmark$ Wound infection

$\checkmark$ Wound gaping

$\checkmark$ Burst abdomen

$\checkmark$ Septicemia,

$\checkmark \quad$ Lung infections like bronchopneumonia

$\checkmark$ Days of hospital stay,

\section{$\checkmark$ Mortality}

2. Postoperative secondary minor procedures like secondary suturing for wound gaping and tension wire banding for burst abdomen and its incidence is also studied.

3. Finally these outcomes are compared between empirical and specific antibiotic administration groups.

\section{Observations and results:}

This study includes 140 patients with perforative peritonitis and who were taken up for surgery and intraoperatively diagnosed to have duodenal perforation. And various data like age, sex, history, clinical examination findings, investigations like routine urine and blood reports, Xray chest PA and abdomen erect view, ultrasonogram of abdomen and pelvis, preoperative diagnosis, intraoperative findings, peritoneal fluid culture and sensitivity report, details of postoperative outcomes in terms of complications like wound infection, gaping, burst abdomen, septicemia, lung infection, mortality, days of hospital stay and no. of cases taken up for secondary minor procedures like secondary suturing and tension wire banding of 140 patients are collected and consolidated .

Then age, sex distribution, the commonest organisms, their sensitive antibiotic pattern, among 140 DU perforative peritonitis patients fulfilling the eligibility criteria , are studied. Also comparison study is done between 70 patients(group I) who were administered with empirical antibiotics and 70 patients(group II) with specific antibiotics according to intraop peritoneal fluid culture and sensitivity report, in terms of post 
operative complications and incidence of postop secondary minor procedures as previously mentioned. The observations and results of the study is depicted in the following pages.

Age distribution:

AGE DISTRIBLTION :

\begin{tabular}{cccc}
\hline AGE & GROLPI & GROLP II & TOTAL \\
\hline$\leq 20$ & 5 & 7 & 12 \\
$21-30$ & 9 & 9 & 18 \\
$31-40$ & 16 & 14 & 30 \\
$41-50$ & 21 & 17 & 38 \\
$51-60$ & 7 & 13 & 20 \\
$61-70$ & 9 & 9 & 18 \\
$>70$ & 3 & 1 & 4 \\
TOTAL & 70 & 70 & 140 \\
\hline
\end{tabular}

Table : 1

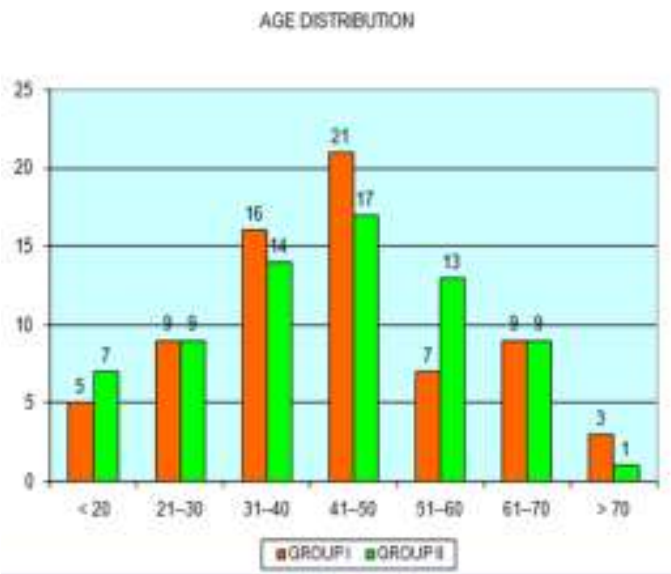

Figure :1

Sex distribution :

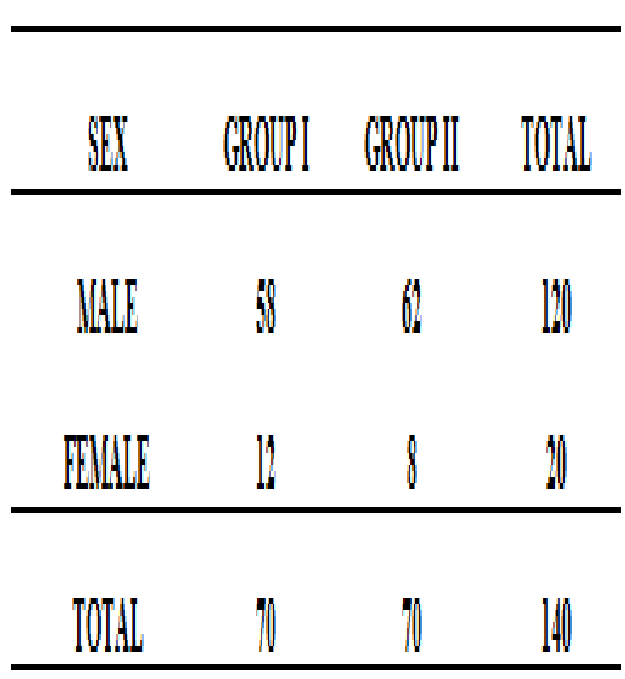

Table: 2

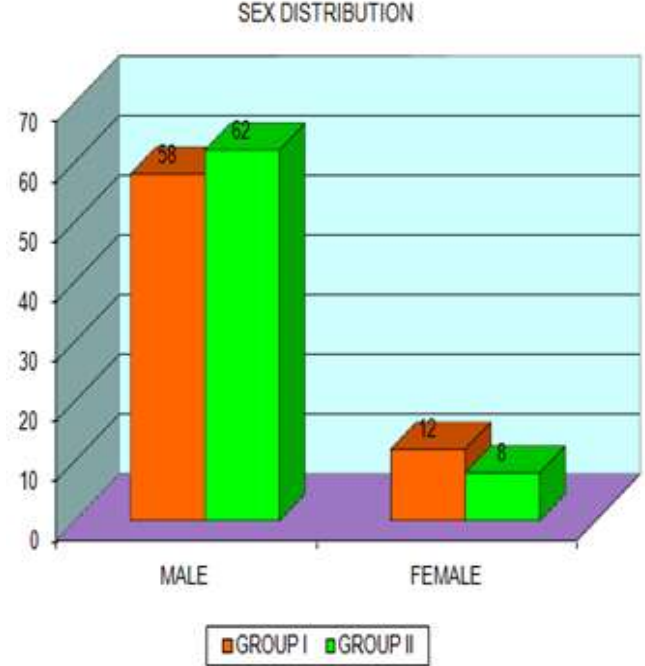

Figure: 2 


\begin{tabular}{cc}
\hline TSOLATED ORGANISMS & TOTAL \\
\hline E.ooli & 63 \\
Klebritll & 27 \\
polymirnobial & 23 \\
Pseubomonas & 10 \\
Streptococci & 8 \\
Staphriboocci & 4 \\
\hline Others & 5 \\
\hline
\end{tabular}

Table:3
ISOLATED ORGANISM DISTRIBUTION

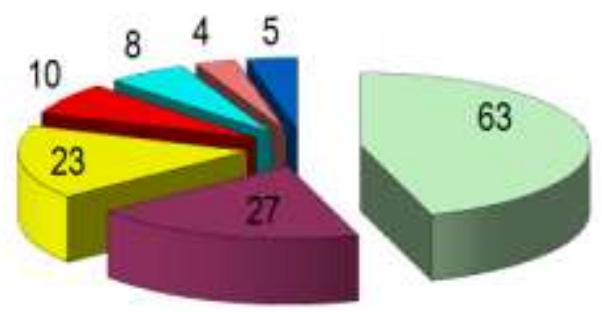

\begin{tabular}{|c|c|c|}
\hline $\begin{array}{l}\text { GE.COLI } \\
\text { Pseudome }\end{array}$ & $\begin{array}{l}\text { Klebsiella } \\
\text { Is aStreptococci }\end{array}$ & $\begin{array}{l}\text { 口polymicrobial } \\
\text { @Staphylococc }\end{array}$ \\
\hline
\end{tabular}

Figure:3

\begin{tabular}{|c|c|}
\hline SENSITVE ANTIBIOTIC & TOTAL: \\
\hline Pigtaz. & 40 \\
\hline Cefotaim & 37. \\
\hline Ceftriawone & 27 \\
\hline Amikacin & 26 \\
\hline Gentartytin & 22 \\
\hline Ciproflatacin & 18 \\
\hline effaptiul & 14 \\
\hline Often & 4 \\
\hline
\end{tabular}

Table: 4

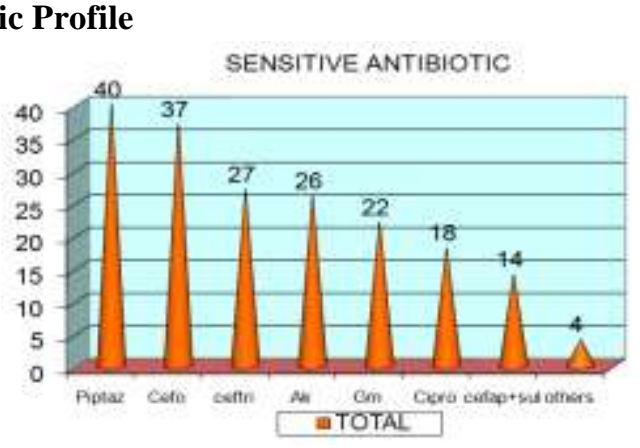

Figure: 4

Fige 4

\begin{tabular}{|c|c|c|}
\hline COMPICATIONS & GROUPI & GROLIP II \\
\hline Woued Infectivn & 39 & 22 \\
\hline Wound Coşing & 18 & 14 \\
\hline Burst Abtomen & 5 & 4 \\
\hline Septicenia & 9 & 5 \\
\hline Lung Infectiva & 9 & 6 \\
\hline Motality & 7 & 3 \\
\hline
\end{tabular}

Table: 5

\section{Postop Outcomes:}

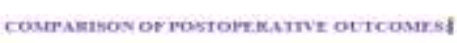

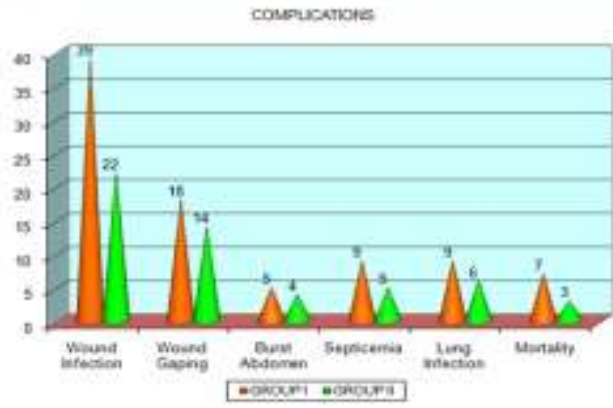

Figure:5

Comparision of postoperative days of hospital stay:

\begin{tabular}{cccc}
\hline $\begin{array}{c}\text { No of days of hospital } \\
\text { stay }\end{array}$ & GROUPI & GROUPI & TOTAL \\
\hline $6-10$ & 30 & 36 & 86 \\
$11-15$ & 32 & 9 & 41 \\
$16-20$ & 7 & 3 & 10 \\
$21-25$ & 1 & 2 & 3 \\
\hline TOTAL & 70 & 70 & 140 \\
\hline
\end{tabular}

Table: 6

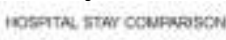

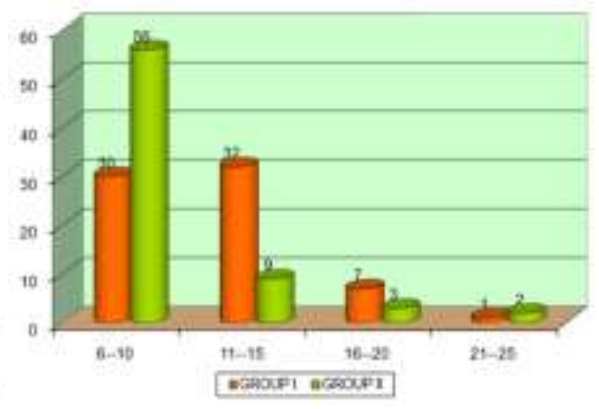

Figure: 6 


\section{Frequency of postop secondary minor procedures:}

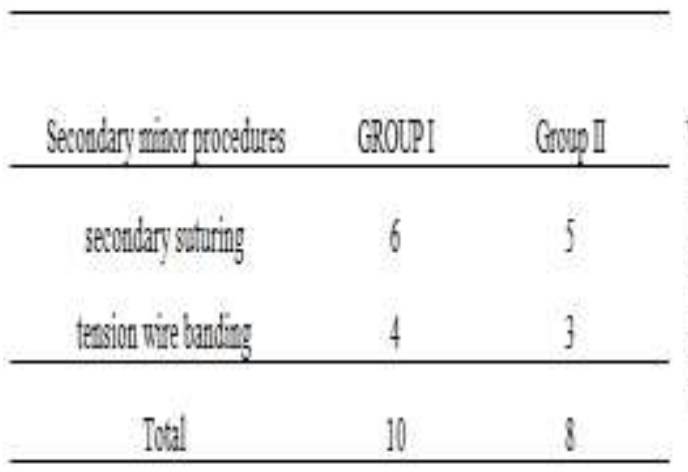

Table: 7

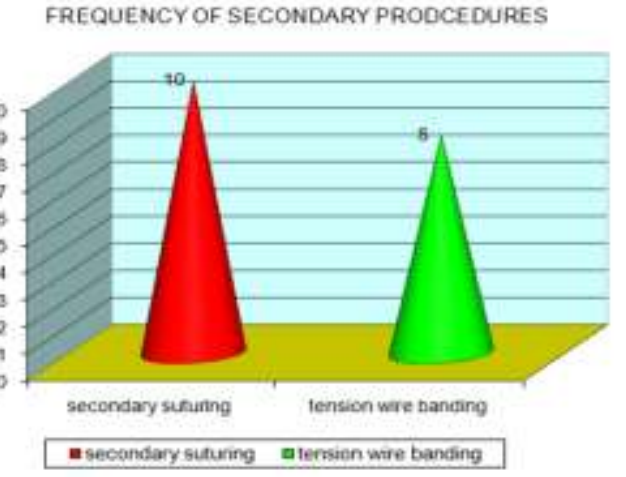

Figure:7

\section{Discussion}

The study includes 140 patients with DU Perforation who fulfills eligibility criteria. Collected informations are consolidated and results are studied. Age distribution has a pyramidal pattern. Peak age group with DU perforation lies in the interval between 41-50 yrs comprising of about $27 \%$ of total study population (38 patients out of 140 patients. Table $1 \&$ Figure 1). Male prepondenrance is observed. 120 out of 140 patients belong to male gender $(85.7 \%$ of the study population are males. Table $2 \&$ Figure 2$)$.

The commonest organism observed in our study is E.coli constitutes $45 \%$ of study population (isolated in 63 out of 140 patients). Others include Klebsiella - 19\% Polymicrobial flora -16\%, Pseudomonas $7 \%$, Streptococcus - 5\%, Staphylococcus-2\%, Other least frequently isolated organisms (3.5\%) include Bacteroides fragilis, Citrobacter, candida etc (Table 3\& Figure 3). One of the recent studies conducted by Punamiya AR, Chougule PG, Ahuja BR et. al., 100 patients with perforated peptic ulcer concluded that E.coli is the most common isolated organisms accounting to $44 \%$. The other organisms constitute $3 \%$, klebsiella $16 \%$. My study results almost have got comparably consistent result with the other studies.

Piperacillin tazobactam and Cefotaxime are found to be the most common antibiotics that is most sensitive against bacteriological flora which are isolated in DU perforative peritonitis. 28.5\% and 26.4\% patients are found to sensitive for piptaz and cefotaxime respectively. Other sensitive antibiotics are ceftriaxome, amikacin, gentamycin, ciprofloxacin, cefaperazone sulbactum, meropenam, imipenam (Table $4 \&$ Figure 4). The study conducted by Punamiya AR, Chougule PG, Ahuja BR et. al. has got piperacillin tazobactam $(51 \%)$ and cefotaxime $(49 \%)$ as highly sensitive drugs in peritoneal fluid culture sensitivity. My study results are almost consistent with this study.

Postoperative complications are compared between group I who were on empirical antibiotics and group II who were on specific antibiotics. 39 pts in group I and 22 pts in group II had wound infection. 18 pts in group I and 14 pts in group II had wound gaping. 5 pts and 4 pts in group I \& II resp. had burst abdomen. 9 pts and 5 pts in group I \& II resp. had septicemia. 9 pts and 6 pts in group I \& II resp. had lungs infection. 7 pts and 3 pts in group I \& II resp expired due to postop complication. The p-value for this comparison is 0.03 which is significant (Table $5 \&$ Figure 5). According to the study conducted by Punamiya AR, Chougule PG, Ahuja BR et. al., the postop complications in group of patients who were administered with specific antibiotic , were significantly less than in patients with empirical antibiotic therapy.

Out of 140 patients , 40\% (56 patients in group II had postop hospital stay of less than 10days) and $21.4 \%$ (30 patients in group I had postop hospital stay of less than 10days). The p-value is 0.037 which is significant (Table 6\& Figure 6). According to Punamiya AR, Chougule PG, Ahuja BR et. al. 25.71\% of patients with empirical antibiotic therapy and $63.33 \%$ of patients with specific antibiotic therapy based on culture sensitivity had hospital stay of less than 10days. Also in that study it is stated that $61.43 \%$ with empirical antibiotic treatment and $26.67 \%$ with specific antibiotic treatment had hospital stay of more than 10days. My study results also found to be consistent with the study conducted by Punamiya AR, Chougule PG, Ahuja BR et. Al. In this study, it is observed that frequency of secondary minor procedures like secondary suturing for wound gaping and tension wire banding for burst abdomen is slightly less in patients who were on specific antibiotics than who were on empirical antibiotics ( Table $7 \&$ Figure 7).

\section{Conclusion}

It is concluded that specific antibiotic administration according to intraoperative peritoneal toxic fluid culture and sensitivity report rather than empirical antibiotic administration, will significantly reduce the 
postoperative outcomes in terms of complications like wound infections, wound gaping, burst abdomen, septicemia, lung infections, mortalities, prolonged hospital stay, increased frequency of secondary minor procedures like secondary suturing and tension wire banding. Since few studies are conducted regarding this aspect, many research works needed to be intiated pertaining to the aspect of administration of specific antibiotic therapy, in different high volume tertiary institutions to validate the use of specific antibiotic therapy rather than using empirical therapy.

\section{References}

[1]. Dale M. Mosdell, M.D., Don M. Morris, et al. 1991.Antibiotic Treatment for Surgical Peritonitis, Dept. of New Mexico: Ann Surg, 214 ( 5 ) : 543-549

[2]. Soares-Weiser K, Brezis M, Leibovici L. 2001.Antibiotics for spontaneous bacterial peritonitis in cirrhotics.Cochrane Database Syst

[3]. Punamiya AR, Chougule PG, Ahuja BR et. al. Commonest organisms and antibiotic sensitivity in peritonitis due to duodenal ulcer perforation in Krishna hospital, Karad. Int J Health Sci Res. 2014;4(8):93-97.

[4]. René G Holzheimer. Surgical Treatment: Evidence-Based and Problem-Oriented. http://rene- holzheimer.de/ Medical Faculty, Martin Luther University Halle-Wittenberg, Halle, Germany.

[5]. Ramakrishnaiah VPN, Chandrakasan C, Dharanipragadha K, Sistla S, Krishnamachari S. Surgical Gastroenterology. DOI: http://dx.doi.org/10.7869/tg.2012.70.

[6]. Nathens AB. Relevance and utility of peritoneal cultures in patients with peritonitis. Surg Infect (Larchmt). 2001 Summer;2(2):15360; discussion 160-2.

[7]. Shrestha K, Paudel BR, Shah LL, Mukhia R, Dahal P, Haque MA, Maharjan SB, Choudhary J. Spectrum of perforation peritonitis 260 cases experience. Postgraduate Medical Journal of NAMS. 2010, 10(2):29-32.

[8]. Afridi SP, Malik F, Rahman SU, Shamim S, Samo KA. Spectrum of perforation peritonitis in Pakistan: 300 cases Eastern experience. World Journal of Emergency Surgery 2008, 3:31.

[9]. Prakash A, Sharma D, Saxena A, Somashekar U, Khare N, Mishra A, Anvikar A. Effect of Candida infection on outcome in patients with perforation peritonitis. Indian J Gastroenterol. 2008 May- Jun;27(3):107-9. 109 Indian Journal of Basic and Applied Medical Research; March 2015: Vol.-4, Issue- 2, P. 105-110

[10]. Shinagawa N, Tanaka K, Mikamo H, Watanabe K, Takeyama H. et al Bacteria isolated from perforation peritonitis and their antimicrobial susceptibilities. pn J Antibiot. 2007 Aug;60(4):206-20.

[11]. Gupta S , Kaushik R. Peritonitis - the Eastern experience . World Journ of Emerg Surgery 2006, 1:13

[12]. H van der Plas. Microbiological evaluation and antimicrobial treatment of complicated intra- abdominal infections. South Afr J Epidemiol Infect 2012;27(2):53-57. 